\title{
Addressing cancer disparities among American Indians through innovative technologies and patient navigation: the walking forward experience
}

\author{
Daniel G. Petereit ${ }^{1,2 *}$, B. Ashleigh Guadagnolo ${ }^{3}$, Rosemary Wong ${ }^{4}$ and C. Norman Coleman 4 \\ 1 Department of Oncology, John T. Vucurevich Cancer Care Institute, Rapid City, SD, USA \\ 2 Department of Human Oncology, University of Wisconsin School of Medicine and Public Health, Madison, WI, USA \\ ${ }^{3}$ Department of Radiation Oncology, The University of Texas M.D. Anderson Cancer Center, Houston, TX, USA \\ ${ }^{4}$ Radiation Research Program, Division of Cancer Treatment and Diagnosis, National Cancer Institute, Rockville, MD, USA
}

\section{Edited by:}

Anatoly Dritschilo, Georgetown

University School of Medicine, USA

Reviewed by:

Stella C. Lymberis, NYU Langone

School of Medicine, USA

Arnold Malcolm, Vanderbilt University

Medical Center, USA

${ }^{*}$ Correspondence:

Daniel Grant Petereit,

John T. Vucurevich Regional Cancer

Care Institute,

353 Fairmont Blvd.

Rapid City, SD 57701, USA.

e-mail:dpetereit@regionalhealth.com
Purpose/Objective(s): American Indians (Als) present with more advanced stages of cancer and, therefore, suffer from higher cancer mortality rates compared to non-Als. Under the National Cancer Institute (NCl) Cancer Disparities Research Partnership (CDRP) Program, we have been researching methods of improving cancer treatment and outcomes since 2002, for Als in Western South Dakota, through the Walking Forward (WF) Program. Materials/ Methods: This program consists of (a) a culturally tailored patient navigation program that facilitated access to innovative clinical trials in conjunction with a comprehensive educational program encouraging screening and early detection, (b), surveys to evaluate barriers to access, (c) clinical trials focusing on reducing treatment length to facilitate enhanced participation using brachytherapy and intensity modulated radiotherapy (IMRT) for breast and prostate cancer, as Als live a median of 140 miles from the cancer center, and (d) a molecular study (ataxia telangiectasia mutated) to address whether there is a specific profile that increases toxicity risks. Results: We describe the design and implementation of this program, summary of previously published results, and ongoing research to influence stage at presentation. Some of the critical outcomes include the successful implementation of a community-based research program, development of trust within tribal communities, identification of barriers, analysis of nearly 400 navigated cancer patients, clinical trial accrual rate of $10 \%$, and total enrollment of nearly 2,500 Als on WF research studies. Conclusion: This NCl funded pilot program has achieved some initial measures of success. A research infrastructure has been created in a community setting to address new research questions and interventions. Efforts underway to promote cancer education and screening are presented, as well as applications of the lessons learned to other health disparity populations - both nationally and internationally.

Keywords: patient navigation, American Indians, cancer disparities, radiation oncology

\section{INTRODUCTION}

In the most recent report (2009) to the nation on the status of cancer by the Centers for Disease Control (CDC), cancer mortality rates declined for all racial and ethnic groups, except American Indians (AI) and Alaskan Natives for whom cancer death rate trends remained level. Unfortunately, these groups did not experience the same annual 1.6\% decrease in mortality from 1999 to 2006 (Espey et al., 2007). AIs who live in the Northern Plains (NP) suffer ageadjusted mortality rates that are significantly higher than those for whites for cancers for which an effective screening test exists. The NP encompass a large geographic swath from Indiana to Montana and includes the Dakotas where a majority of Lakota Sioux live (Figure 1). The cancer-specific mortality rates for NP AIs are 79\% higher for cervical cancer, 58\% higher for colorectal cancer, and $49 \%$ higher for prostate cancer (Espey et al., 2003).

AIs utilize cancer screening at much lower rates compared to the national average (Ward et al., 2004). As a result, NP AIs present with disparately high rates of advanced-stage cancer
(Petereit et al., 2004; Rogers and Petereit, 2005). In 2003, a review of the Rapid City Regional Hospital (RCRH; Rapid City, South Dakota) tumor registry (1990-2000) revealed that $50 \%$ of AI cancer patients presented with stage III-IV breast, colorectal, prostate, cervical, and lung cancer compared with $36 \%$ of nonAmerican Indians presenting with advanced stage disease for those malignancies (Petereit et al., 2004). In the same region there are non-AI cancer patients who are medically underserved as well - many who live in remote rural locations. Root causes for these disparities are multiple, and there is a growing literature dedicated to examining and solving the patient-, physician-, and health-care system related factors that contribute to inequities in health outcomes by race and ethnicity (Lannin et al., 1998; Geiger, 2003).

AI populations have valid reasons for distrust of researchers (Burhansstipanov et al., 2009). Christopher et al. (2005) documented multiple examples of Natives' distrust of researchers based on numerous historical events. Unethical research or care protocols 


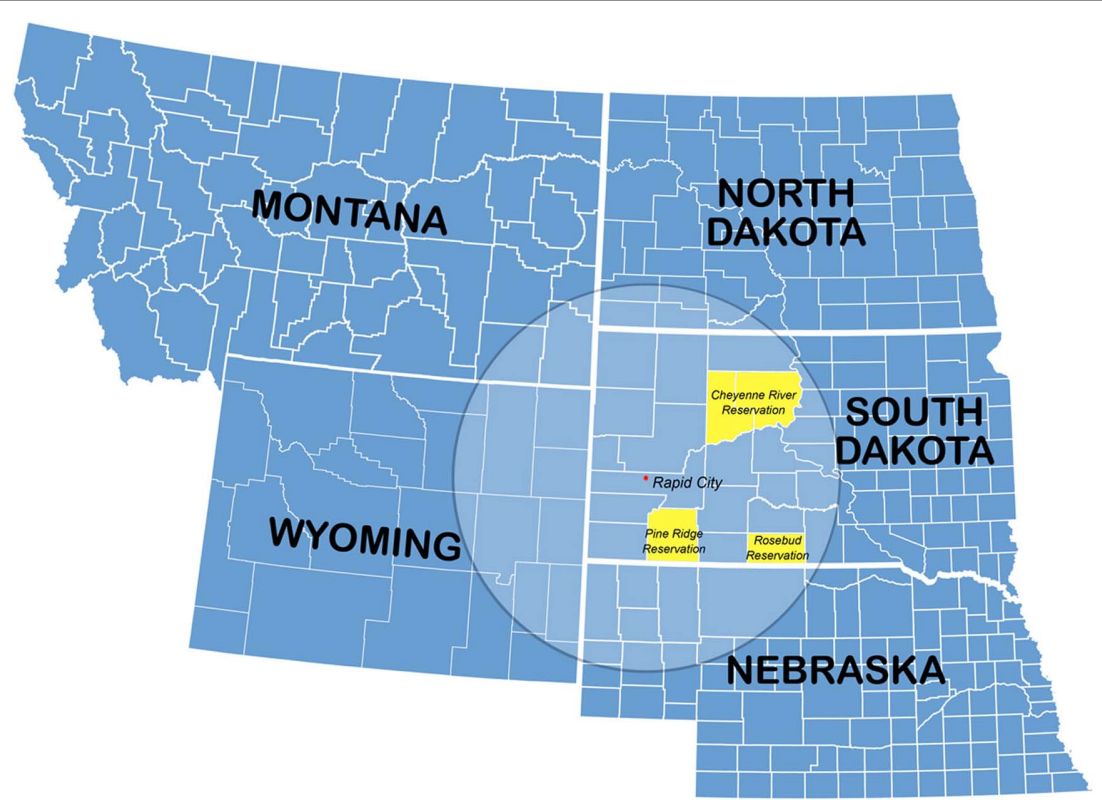

FIGURE 1 | Geographic area served by Walking Forward (radius shown 180 miles).

have historical relevance to government policies designed to annihilate AIs in the nineteenth and the first half of the twentieth centuries; others are more recent and include sterilization of AI women without informed consent in the 1970s (Burhansstipanov et al., 2009). Most recently, Arizona State University (ASU) agreed to pay $\$ 700,000$ to 41 members of the Havasupais Indian tribe to settle legal claims that university researchers improperly used tribe members' blood samples in genetic research (Mello and Wolf, 2010). Although the initial tragedy of Wounded Knee (Pine Ridge, SD) occurred over 100 years ago (1890), when honoring those who died in 1990, racist violent attacks were again made against the Natives in this region. Thus, these and many other historical trauma events are fresh in the memory of AIs living in SD. Appreciating and understanding the multiple reasons for this lack of trust is critical in order for researchers to pursue areas of investigation in this population (Petereit and Burhansstipanov, 2008; Burhansstipanov et al., 2009).

The National Cancer Institute (NCI) created the Cancer Disparities Research Partnership (CDRP) pilot program in 2002 to reduce cancer disparities observed among underserved sections of the United States population. RCRH was one of six sites to receive the grant. RCRH provides secondary and tertiary cancer care for approximately 60,000 adult AIs living in nearby reservations, surrounding rural communities, and Rapid City itself. The three reservations served include Pine Ridge, Rosebud, and Cheyenne River (Figure 1). The geographic area is approximately 100,000 square kilometers. The $1 n$ order to address the ominously high-cancer death rates for NP AIs, a multifaceted program called Walking Forward (WF) was developed through NIH funding (PI Petereit D). The Cancer Care Institute (CCI) is located within RCRH which is the central location for WF. This program will be further described and includes assessment of cancer barriers, clinical trials, radiogenomics, and patient navigation.
Patient Navigation (PN) has emerged as a new strategy to address cancer disparities, and in our region to address issues of medical mistrust. In the early 1990's Freeman and colleagues in Harlem demonstrated improved 5-year survival from 39 to $70 \%$ for breast cancer patients that underwent their model of patient navigation (Freeman et al., 1995; Freeman, 2006). As part of the NCIs CDRP program, each site was to implement PN tailored to their respective population.

Clinical trial participation is critical as it provides patients access to state-of-the-art therapy; safe and effective treatments that are closely monitored; and the potential for improved clinical outcomes, including quality of life. The rate of accrual in clinical trials among minority, rural, and lower socioeconomic subpopulations is low in the US and is approximately 1\% (Maurer et al., 2001; Sateren et al., 2002; Murthy et al., 2004). This may be a result of numerous barriers to accessing health care that could potentially exacerbate known cancer-related health disparities among underserved populations. (Shavers and Brown, 2002; Petereit et al., 2004; Espey et al., 2007) The low participation rates among minorities also limit the treatment implications of data derived from clinical trials. As part of the NCI CDRP initiative, clinical trial development tailored to these special populations, and potential enrollment, was a critical outcome.

While the emphasis of this article is on the WF program, we will also describe how the "lessons learned" in this population may be applied to other disparate populations in the US and abroad.

\section{MATERIALS AND METHODS PRIMARY HYPOTHESIS}

AIs in western, SD, live a median of 140 miles from the cancer center in Rapid City (Figure 1). The identification of barriers which prevent AIs from presenting with earlier stages of cancer, or in some circumstances not at all, was investigated. It was hypothesized that 
a major barrier to obtaining timely and effective cancer treatment was distance from the cancer center as conventional radiotherapy requires 6-8 weeks. Therefore, phase II studies were developed to address this potential barrier for malignancies commonly seen among the AIs, breast cancer and prostate cancer, utilizing intensity modulated radiotherapy (IMRT) and brachytherapy. At the community level, we engaged in several social science studies to determine potential barriers to cancer screening and early detection. In addition, our community staff was responsible for outreach, education, and networking.

\section{COMMUNITY-BASED PARTICIPATORY RESEARCH}

In 2001, the NCI released a Request for Application (RFA) requesting planning grant applications from communities that experience health disparities (Table 1). The PI coordinated meetings with both the tribal leadership and Indian Health Service (IHS) to introduce the general principles of cancer treatment and cancer research studies and to present the RFA for their consideration. The sentinel issues raised were trust and levels of commitment from physicians and researchers, high-cancer death rates, and potential resolutions. After much discussion, the tribal councils approached were in favor of partnering with RCRH on the research proposal. They prepared and passed a tribal resolution in support of the study and included letters of support (Petereit and Burhansstipanov, 2008).

Once the grant was rewarded in 2002, the PI returned to each reservation for further discussion and implementation. Since the tribal councils are elected every other year, it was critical to maintain a constant level of dialog for ongoing approval for WF as many of the research studies were conducted within the community. Many tribal members and leaders were rightfully suspect that our project would be another "fly by night" operation where we would come in, abstract the data, leave, and publish the results without any feedback or continuity (a phenomenon commonly referred to as "helicopter research" by many Native communities). Thus, quarterly reports were presented to the tribal councils so they could monitor ongoing research - both at the community level and at the cancer center.

\section{IRB APPROVAL}

The three reservations in western, SD, are considered sovereign nations which mandate a more comprehensive IRB approval process. IRB approval was required from the Aberdeen Area IHS (IHS IRB of record for the NP), National IHS, RCRH, the University of Wisconsin (UW), the Mayo Clinic (ataxia telangiectasia mutated, ATM study only) and the NCI. The Aberdeen Area IHS IRB would consider protocols only after the scientific validity was reviewed and approved by the UW IRB, and only after letters of support and/or tribal resolutions were obtained from the service unit director of IHS, tribal councils from each reservation, as well as a letter of support from the Aberdeen Area Tribal Chairmens' Health Board (AATCHB). Therefore, each protocol required seven letters of support and/or resolutions. With 9 initial protocols 72 letters of support were received. Due to the rigorous and necessary peerreview process for our project, it took a minimum of 12 months to activate the first study (Table 1). Consents were written in a culturally appropriate manner with guidance, direct feedback and input from the AI community, often through focus groups. This process

Table 1 | Walking forward timeline.

\begin{tabular}{|c|c|}
\hline Date & Activity \\
\hline Spring 2000 & PI initiates dialog with tribal councils to present the current status of cancer treatment in Rapid City \\
\hline July 2001 & RFA issued \\
\hline Sept 2001 & Meetings with tribal councils to discuss RFA \\
\hline Oct 2001 & Grant preparation \\
\hline March 2002 & Grant submitted \\
\hline Sept 2002 & Grant awarded to Rapid City \\
\hline Sept 2002 - May 2003 & Staff hiring, protocol development \\
\hline January 2003 & Quarterly meetings with tribal councils to discuss implementation of walking forward \\
\hline February 2003 - April 2003 & Presentations to multiple tribal councils, tribal health boards, IHS clinics to obtain letters of support for each protocol \\
\hline June 2003 & First protocol submitted to one 4 IRBs (Patient Navigation Study) \\
\hline January 2004 & Patient navigation protocol activated \\
\hline October 2004 & All 12 protocols activated \\
\hline Sept 2007 & Supplemental funding through $\mathrm{NCl}$ to promote cancer screening through community education \\
\hline May 2008 & $\begin{array}{l}\text { Partnership with Native American Cancer Research Inc. on a project funded by the National Center on Minority Health and } \\
\text { Health Disparities to promote cancer education }\end{array}$ \\
\hline September 2008 & Over 1,800 American Indians enrolled on various WF clinical research trials \\
\hline December 2008 & Rapid City submits competing renewal application to $\mathrm{NCl}$ for continuation of WF program \\
\hline September 2009 & Rapid City receives funding for another 5 years \\
\hline January 2011 & Nearly 2,500 American Indians enrolled on various WF clinical research trials \\
\hline April 2010 & Small award from Potowatami Tribe to promote cancer screening in the community \\
\hline March 2011 & $\begin{array}{l}\text { Comprehensive patient navigation implemented with WF staff embedded within each IHS hospital to facilitate cancer } \\
\text { screening }\end{array}$ \\
\hline November 2011 & $\begin{array}{l}\text { Partnership with South Dakota Department of Health, on a project funded by the CDC, to expand and support existing patient } \\
\text { navigation programs }\end{array}$ \\
\hline
\end{tabular}


has been described in further detail. (Molloy et al., 2007; Petereit et al., 2008) The IRB of record for WF was RCRH, particularly for all research activities that took place at the cancer center in Rapid City. Finally, all manuscripts were reviewed and edited for potential approval by the IHS and RCRH IRBs.

\section{ATAXIA TELANGIECTASIA MUTATED}

It was the subjective experience of CCI radiation oncologists that AIs experience higher rates of radiation toxicities that could represent a treatment barrier as well. A genetic milieu may exist which renders AIs more sensitize to radiation. Therefore, a laboratory study was conducted to determine whether AIs have a higher ATM variant rate compared to non-AIs. The ATM gene was analyzed from both AI and non-AI patients undergoing radiation with prospective scoring of toxicities. The methodology has been previously described (Petereit and Burhansstipanov, 2008; Petereit et al., 2010).

\section{PATIENT NAVIGATION}

A process map for patient navigation was developed to overcome the identified barriers and build trust within the community. Although this model shared similar characteristics to the one developed by Feeman (2004-2006), previous director of the NCI Center to Reduce Cancer Health Disparities, our model was tailored to meet the needs of our population. Our program has been previously published and detailed elsewhere (Petereit et al., 2008; Guadagnolo et al., 2011).

The WF patient navigation program included services for cancer patients and a community outreach program. Cancer patients were assisted by hospital based patient navigators to address barriers and facilitate cancer-related care, encourage clinical trial participation, and follow-up assessment. The community research representatives (CRR), based in their respective communities on each of the three reservations, facilitated an education and outreach component. All of the CRRs were AI and members of the respective tribe they served. These CRRs promoted early detection through screening and raised awareness of the clinical trials by implementing culturally relevant education modules. They also administered a survey to determine the barriers and determinants of cancer screening. A core feature of the navigation program was ensuring cultural competency of the navigators as detailed in a previous publication (Molloy et al., 2007).

\section{IDENTIFYING BARRIERS TO HEALTH CARE (SURVEYS)}

To identify the barriers to timely cancer diagnosis and treatment, a community survey and a cancer patient survey were developed through a collaborative process employing a community-based participatory research (CBPR) methodology. The community survey was implemented among nearly 1,000 AI in the target communities, while the cancer patient survey was implemented prospectively among 165 newly diagnosed cancer patients at RCRH's CCI, of which 52 were AI. The methodologies and results have been previously published (Guadagnolo et al., 2009a,b; Pandhi et al., 2010a,b).

\section{CLINICAL TRIALS}

Clinical trial enrollment data was prospectively tracked for all navigated AI patients. Enrollment of AIs on NCI sponsored clinical trials was a high priority as approximately 75 studies were open. Phase II clinical trials were available for prostate and breast cancer patients utilizing IMRT and brachytherapy with the theme of reducing overall treatment time. Eligible stage I breast cancer patients were offered breast brachytherapy; whereas, prostate patients were offered IMRT \pm brachytherapy depending upon their risk stratification as defined by D'Amico et al. (1998). Details of these phase II trials have previously been described (Petereit et al., 2004; Ritter et al., 2009; Adkison et al., 2010).

\section{SUMMARY OF M AND M}

Through patient education, screening, assessing potential barriers to health care, and enrollment on innovative treatment strategies, it was hypothesized that AIs would eventually present earlier in their disease process, have increased access to NCI sponsored clinical trials, and ultimately experience higher cure rates. In addition, clinical trials were developed to meet the needs of the population served. A central theme was truncation of radiation utilizing advanced technologies.

\section{RESULTS \\ PATIENT NAVIGATION}

Between February 2004 and September 2009, 332 AI cancer patients received PN services throughout cancer treatment. The median number of contacts with a navigator was 12 (range, 1-119). The median time spent with the navigator at first contact was $40 \mathrm{~min}$ (range 10-250 min.) and 15 min for subsequent contacts. Patients treated with radiation therapy with curative intent who underwent PN had fewer days of treatment interruption (mean, 1.7 days; $95 \%$ CI, 1.1-2.2 days) than historical controls who did not receive PN services (mean, 4.9 days; 95\% CI, 2.9-6.9 days; Guadagnolo et al., 2011).

\section{COMMUNITY SURVEY}

Given that most of the AI communities reside in rural locations, distance and transportation present significant barriers to accessing health care as $47 \%$ of the respondents identified lack of transportation as one of the most common barriers to accessing care. For example, $75 \%$ of respondents reported traveling more than 50 miles for tertiary medical care while AIs seeking cancer treatment at RCRH traveled an average of 140 miles one way (Guadagnolo et al., 2009b; Pandhi et al., 2010b).

The respondents also identified lack of satisfaction in interactions with medical providers and a lack of confidence in their abilities as additional barriers to care. AI cancer patients exhibited a significantly higher mistrust $(p=0.0001)$ and lower satisfaction ( $p=0.0001$ ) with health care than whites (Guadagnolo et al., 2008).

Additional barriers specific to accessing cancer-related health care, including cancer screening were also identified. Overall, only $43 \%$ of participants reported ever having had any cancer screening tests. Analysis of cancer-specific screening rates showed the following percentages: cervical $48 \%(n=596)$, breast 59\% $(n=369)$, prostate $34 \%(n=115)$, and colorectal $20 \%(n=56)$. These rates are significantly lower than the published rates for not only US nonHispanic Whites, but also for AI, specifically the NP AI population (Espey et al., 2007; Pandhi et al., 2010b). There were several significant determinants of cancer screening prevalence. Most importantly, those who reported that a doctor or nurse had recommended cancer screening had nearly 12 times the odds of ever receiving screening (OR 11.99; 95\% CI 7.35-19.56; Pandhi et al., 2010b). 


\section{CANCER SURVEY}

There was a significant difference in mean scale scores for medical mistrust by race. In the univariate analysis, AIs exhibited higher mean scores for mistrust than Whites $(p=0.0001)$. A multivariable regression model including race, age, gender, employment status, annual income, education level, and distance from CCI revealed that AIs exhibited a significantly higher level of mistrust ( $p=0.008)$, even when adjusting for the other variables. No other variable was associated with a significant difference in mistrust scores in the multivariable model (Guadagnolo et al., 2009a).

There was also a significant difference in mean scale scores by race for satisfaction with the health care system and providers. AI patients had lower mean scale scores for satisfaction than did White patients $(p=0.0001)$. In a multivariable model analyzing satisfaction mean scale score showed that AIs had significantly lower scores for satisfaction with health care than Whites $(p=0.0001)$, even after adjusting for age, gender, employment status, income, education level, and distance from CCI (Guadagnolo et al., 2009a).

\section{CANCER EDUCATIONAL WORKSHOPS AND SCREENING}

In the last 3 years, cancer education efforts were initiated with the goal of increasing screening rates by $20 \%$. Cancer screening educational workshops were conducted as an educational intervention. A total of 410 individuals participated in cancer screening workshops. Participants demonstrated significant increases in cancer screeningrelated knowledge levels with an increase in knowledge quiz score of $21 \%$ in cervical cancer workshops $(p<0.001), 24 \%$ in breast cancer workshops $(p<0.001), 21 \%$ in prostate cancer workshops $(p<0.001)$, and $22 \%$ in colorectal cancer workshops $(p<0.001)$. We are currently following up on all participants eligible for cancer screening to determine any increase in the screening rates post-workshop. To date we have followed up on 125 participants eligible for screening and preliminary data indicates an increase of screening rate for breast, cervical, and prostate cancer, but not colorectal cancer (Subrahmanian et al., 2011).

\section{PHASE II CLINICAL TRIALS}

Forty-two non-AI and 5 AI patients have been enrolled on the breast brachytherapy study with ongoing accrual. The low-risk IMRT hypofractionated prostate study has enrolled 307 patients among 5 institutions nationally, with 43 enrolled from Rapid City (4 AIs). Preliminary results have been published with excellent biochemical control and acceptable toxicities (Ritter et al., 2009). This study continues to accrue patients, and is one of the most successful trials ever opened in Rapid City in regards to treatment tolerance, patient satisfaction, and accrual. Eight patents (1 AI) have enrolled on our intermediate-risk study investigating a short course of IMRT followed by a permanent seed implant boost, and 53 patients ( $1 \mathrm{AI}$ ) have been enrolled on the high-risk prostate study, in conjunction with UW, which investigates hypofractionated radiation to both the prostate and pelvic lymph nodes. Preliminary results from the high-risk study were recently published (Adkison et al., 2010).

\section{ATAXIA TELANGIECTASIA MUTATED}

We recently reported preliminary ATM sequencing data for $100 \mathrm{AI}$ and 100 non-AI patients (Petereit et al., 2010). There were no statistically significant differences for total prevalence of SNPs among AI $(40 \%)$ and non-AI $(48 \%)$ patients $(p=0.32)$. We are currently correlating SNP data to clinical toxicities to assess a potential relationship. While there were initial concerns that this study could not be opened, or accrued to, due to concerns of genetic testing in a disparate population, IRB approval was rapidly obtained with only a few patients refusing participation. Patients were eager to enroll as they believed it could help the next generation of cancer patients.

\section{NCI SPONSORED CLINICAL TRIALS}

During the WF era (2002-present), 358 patients were enrolled on NCI studies, including 32 AIs. When these numbers are combined with the above investigator initiated trials, the total number of patients accrued was 619, of which 145 were AI. We have enrolled nearly 2,500 AIs on various WF research studies. While the majority of enrolled participants were on social science investigations, these studies all required IRB approval, individual informed consents, and in essence, validated the trust that was established within each community / reservation.

\section{REASONS FOR LACK OF ACCRUAL}

Between September 2006 and January 2008, 891 new cancer patients (AI and non-AI) were evaluated for potential participation in a clinical trial. Seventy-eight patients (9\%; 95\% CI, 7-11\%) were enrolled on a clinical treatment trial. For 73\% (95\% CI, 69-75\%) of patients (646 of 891) lack of relevant protocol availability or protocol inclusion criteria restrictiveness was the reason for non-enrollment. Only 45 (5\%; 95\% CI, 4-7\%) patients refused enrollment on a trial. Of the 78 enrolled on a trial, 6 (8\%; 95\% CI, 3-16\%) were AI. Further details of this analysis have been published (Guadagnolo et al., 2009c).

\section{DISCUSSION}

Walking Forward is a multifaceted cancer disparity program that has achieved some initial level of success including establishment of trust within the AI community, development of research infrastructure in a community hospital, high enrollment of AIs on various research protocols, implementation of advanced radiation technologies, and the creation of a comprehensive patient navigation program. The process of initiating, nurturing and establishing a trusting partnership with any underserved community must occur at multiple levels, beginning with shared participation in the planning processes, continued consultation through project implementation, and sharing responsibilities for data analyses and writing and dissemination of study findings. Establishing a trusting partnership with the community takes time and is the foundation of CBPR, particularly with the AI population where historic trauma and distrust of researchers is pervasive. Our patient navigation program was key to establishing trust at the community level which enhanced cancer care and clinical trial enrollment at the cancer center in Rapid City as detailed in Table $\mathbf{1 .}$

Patient navigation is a health access barrier-focused intervention becoming increasingly adopted as a health care delivery innovation to address disparate cancer-related health outcomes. Such disparities are well-documented among minority and low income patients who often face multiple health access barriers ranging from financial and logistical constraints in obtaining health care to cultural and trust issues that impede health care provision (Guadagnolo et al., 2011). 
Patient Navigation was one of our key strategies to address complex patient issues among the AIs. Much of the PN literature addressing care of cancer patients focuses on increasing screening incidence and/or decreasing intervals between screening and diagnostic management of initiation of treatment for various cancers (Weinrich et al., 1998; Dignan et al., 2005; Nash et al., 2006; Christie et al., 2008; Wells et al., 2008), and most studies do show improvement in the corresponding outcomes when PN is available. AIs specifically have been shown to benefit from PN in the setting of facilitating screening and timely diagnostic follow-up (Burhansstipanov et al., 2000; Dignan et al., 2005). However, few studies report upon the role of PN after a patient is diagnosed and undergoing the rigors of cancer treatment (Dohan and Schrag, 2005; Wells et al., 2008), arguably a time when patients may experience more barriers due to logistical complexities and financial burden of receiving many weeks or months of treatment, e.g., RT or chemotherapy.

Our finding of fewer treatment interruptions for patients undergoing curative RT is one that has important implications for this population that has been shown to have disparately highcancer mortality. (Rades et al., 2008; McCloskey et al., 2009) This is especially relevant for treatment of cervical cancer - a disease that disproportionately affects poor and minority women, and for which AI women in the NP have the highest incidence and mortality (Petereit et al., 1995; Espey et al., 2007; Haverkamp et al., 2008). Patient navigation, in our program, seemed to offer an effective strategy to mitigate barriers that may prolong definitive cancer treatment.

Multiple studies have indicated that clinical trial participation among AI cancer patients is low with trial accrual rates of $<1 \%$ (Sateren et al., 2002; Murthy et al., 2004; Stewart et al., 2007). Reasons underpinning low minority participation in clinical trials are multi-factorial and may differ across regions as well as between racial/ethnic groups (Bruner et al., 2006; Ford et al., 2008). In this region, it has previously been shown that AI cancer patients exhibit relatively high rates of medical mistrust and dissatisfaction with prior health care received (Guadagnolo et al., 2009a). Our PN program specifically targets health access barriers in this population and seeks to address them in a culturally sensitive manner while partnering with tribal communities. This effort toward establishing rapport with AI patients and communities to meet their needs may account for our relatively higher proportion of AI patients willing to enroll on clinical trials.

A primary reason for clinical trial ineligibility is advanced stage/ metastasis at presentation. To address this stage disparity, we have embarked upon community education to enhance cancer awareness for screen detectable cancers on all three reservations. Over $600 \mathrm{AI}$ community members have now participated in these educational events with pre and post-testing demonstrating improved knowledge. If interested, these participants are referred to our cancer screening coordinators (CSCs) who work within the IHS clinic/ hospital on each reservation and in Rapid City to arrange for cancer screening (Figure 2). If patients are diagnosed with cancer, they are eventually referred to the cancer navigators in Rapid City who are part of the cancer team. To date, 272 AIs have undergone cancer screening as a result of these educational workshops. In addition, our community navigation program coordinates cancer screening events by networking with other organizations such as the Susan

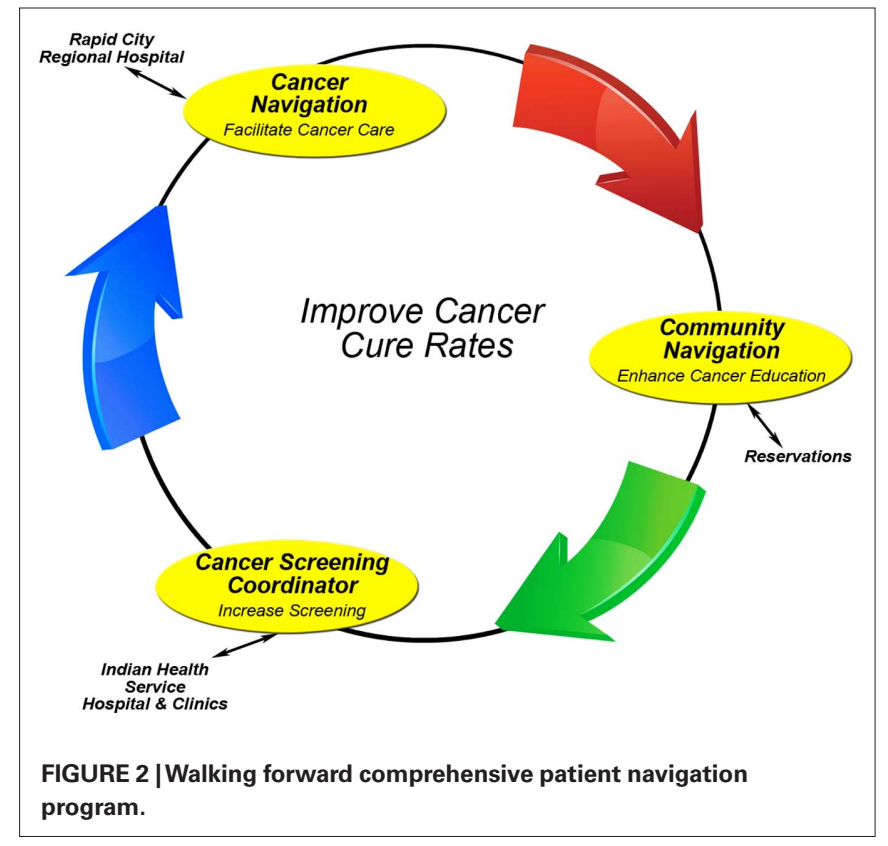

Komen Foundation, the American Cancer Society, a CDC funded colorectal project, the regional radiologists and other groups that perform cancer screening. Through these expanded screening interventions, it is the long-term goal of WF to lower cancer mortality rates by influencing stage at presentation, as well as increasing clinical trial accrual.

\section{CLINICAL TRIALS}

Clinical trial enrollment is the cornerstone of advancing cancer care. Although many clinical trials were available through the cooperative group mechanism, many of these studies had eligibility criteria that were too restrictive, or did not meet the needs of our population. Therefore, clinical trials were developed for common cancers observed in the AI and non-AI population that addressed their unique needs.

A 1992-1993 Medicare survey reported that only $1.4 \%$ of Medicare patients underwent breast conservation in Rapid City, SD (Wennberg, 1996; Kanekar and Petereit, 2009). Although the reasons are many, the distance from the cancer center is presumed to be a major impediment to breast conservation. The inverse relationship between breast conservation rates and the distance from the cancer center is supported by the observation of lower rates of breast preservation in rural areas (Nattinger et al., 1992; Wennberg, 1996). Therefore, our phase II breast brachytherapy protocol was activated to address the unique needs of our patients. To date, no patients have locally recurred and cosmetic outcomes have been good to excellent with followup and further enrollment continuing. Breast brachytherapy has expanded the options for breast preservation for patients in western, SD, as many patients live $2-3 \mathrm{~h}$ from the cancer center in Rapid City.

The incidence of prostate cancer for AI men parallels the incidence of US men as the most common malignancy. Three phase II clinical trials were implemented for men with low-, 
intermediate-, and high-risk prostate cancer (non-metastatic). A central theme to these studies was the use of hypofractionation to exploit a potential radiobiologic advantage and to decrease the overall treatment time. The low-risk prostate study was one of the most successful trials opened in Rapid City as we accrued nearly 50 patients and the trial met the needs of our population. Nationally, this trial has accrued over 300 patients with excellent biochemical control rates, but with late G2 rectal bleeding rates of $8 \%$ which have all resolved. Implications for this are discussed below in the ATM section.

There is currently a need in the US to offer patients participation in clinical trials that address both special needs and a relevant, oncologic issue. For example, women in our part of the country had a low accrual rate to the phase III NSABP B-39 /RTOG 0413 trial $(n=4)$; whereas, women were eager to participate in our phase II breast brachytherapy trial $(n=47)$. Many of these women trial indicated they would choose a mastectomy over 6 weeks of external beam radiotherapy due to the distance from the cancer center. Thus, the needs of these special populations should be considered among the cooperative groups in the design of future phase II, clinical trials for institutions that serve these populations. Themes worthy of investigation include hypofractionation, dose escalation, radiosensitizers, minimization of toxicities and resource sparing. Hypofractionation currently remains investigational, but if validated by ongoing trials, the potential exists for a significant therapeutic gain, as well as substantial health care cost and utilization advantages. These trials will have significant implications for radiation delivery in resource limited countries.

\section{RADIOGENOMICS}

As part of the original research design, the ATM gene was investigated as there was concern that the AI patient population was more sensitive to the effects of therapeutic radiation. Fear of this treatment related side effect could potentially contribute to the commonly observed treatment delays observed in this patient population. To date, $100 \mathrm{AI}$ and 100 non-AI participated in the ATM study (Petereit and Burhansstipanov, 2008; Petereit et al., 2010). This study provided new information on the ethnic distribution of ATM SNPs in a regional US population, and was the first to determine the prevalence of ATM SNPs in AIs. Correlating the SNP data with available patient toxicity data is underway and may reveal any important SNPs that can be predictive of radiosensitivity. There is no support in our study for the hypothesis that a difference in prevalence or type of ATM SNPs explains the different spectrum of side-effects seen after radiotherapy in AI versus non-AI patients.

As an extension of the ATM study, we are investigating the genetic risk profiles of individuals by identifying genes through DNA microarrays that might predict response to treatment, and ultimately, guide therapy. The ultimate goal is to identify markers that could be used to identify patients that might have an adverse response prior to treatment and to determine whether the causes of susceptibility of AI and other populations are similar or different. This is particularly relevant in our population as data continues to mature that supports the role of hypofractionation in a number of clinical scenarios; however, if patients exhibited a molecular profile of increased radiosensitivity, than these strategies would not be contraindicated.

\section{BUILDING FROM SUCCESS}

Some of the lessons learned were predictable, the experience was challenging yet invigorating and a number of the observations are profound. It was predictable that distance from the treatment center, transportation, family and work obligations, and financial considerations adversely impacted cancer care. That the stage at diagnosis was advanced was expected but that there was such a paucity of clinical trials within the NCI portfolio was an excellent lesson learned. Indeed, some of the treatment regimens tailored to advanced disease and limited duration of therapy for CDRP are applicable worldwide for disparities populations. That it would require effort to build trust was expected, but the desire on the part of the community and AIs to indeed build trust was very strong. The supposition by some that AIs would never agree to tissue biomarker studies was wrong. That people would readily cross a range of barriers to fight the common enemy of disease was uplifting and the spread of AI self-advocacy from cancer care to other aspects of health care as a result of CDRP and WF is a terrific spinoff. The struggle to sustain the program was an issue and remains a serious challenge. Appropriately, some program reviewers said "we were taking on the toughest challenges." We agreed and argue that if you can solve the toughest ones, you can simultaneously solve many easier ones. This program was granted a final 5-year period in 2009 so new sources of support will be needed.

Perhaps the most profound lesson is the importance of effort, caring, listening, and learning from one another and the good that comes to both sides of the provider-patient equation. The progress made by WF and all of those involved emphasizes how much a small number of dedicated individuals who are trying to make a difference can really make.

"Never doubt that a small group of thoughtful, committed citizens can change the world; indeed, it's the only thing that ever does" Margaret Mead (http://thinkexist.com/quotation/never_doubt_ that_a_small_group_of_thoughtful/199313.html).

A key lesson learned is the value of person-to-person relationships and how such trust, caring, and effort can be effective. Sadly, there are few opportunities for this community/altruistic type of work within a medical career, a gap we hope to fill with a new concept the Medical Expert Corps and Cancer Expert Corps: The Peace Corps for Cancer to address health disparities for chronic diseases. That is a work in progress and the success of WF demonstrates the multiplier effect of such efforts (Reynolds, 2010).

\section{CONCLUSION}

Walking Forward is a community-based program that has achieved several measures of success including establishment of trust within the AI population, unprecedented rates of clinical trial accrual, and creation of a research infrastructure that permits us to address ongoing research questions of relevance to this community. Through a comprehensive patient navigation program a reduction in cancer mortality is anticipated as AIs will present with earlier stages of cancer, and therefore, will have increase eligibility for access to clinical trials.

\section{ACKNOWLEDGMENT}

Work funded by NIH grant - RFA 1U56CA99010-01. 


\section{REFERENCES}

Adkison, J. B., McHaffie, D. R., Bentzen, S. M.,Patel, R. R., Khuntia, D., Petereit, D. G., Hong, T. S., Tomé, W., and Ritter, M. A. (2010). Phase I trial of pelvic nodal dose escalation with hypofractionated IMRT for high-risk prostate cancer. Int.J. Radiat. Oncol. Biol. Phys. doi: 10.1016/j.ijrobp.2010.09.018. [Epub ahead of print].

Bruner, D. W., Jones, M., Buchanan, D., and Russo, J. (2006). Reducing cancer disparities for minorities: a multidisciplinary research agenda to improve patient access to health systems, clinical trials, and effective cancer therapy. J. Clin. Oncol. 24, 2209-2215.

Burhansstipanov, L., Bemis, L. T., and Petereit,D.G. (2009). “Native American communities: perspectives on healthcare and genetics", in Genetics and Ethics in Health Care: New Questions in the Age of Genomic Health, ed. R. Monsen (Silver Spring, MD: American Nurses Publishing), 179-199.

Burhansstipanov, L., Dignan, M. B., Bad Wound, D., Tenney, M., and Vigil, G. (2000). Native American recruitment into breast cancer screening: the NAWWA Project. J. Cancer Educ. 15, 29-33.

Christie, J., Itzkowitz, S., Lihau-Nkanza, I., Castillo, A., Redd, W., and Jandorf, L. (2008). A randomized controlled trial using patient navigation to increase colonoscopy screening among lowincome minorities. J. Natl. Med. Assoc. 100, 278-284.

Christopher,S. (2005). Recommendations for conducting successful research with Native Americans. J. Cancer Educ. 20, 47-51.

D'Amico, A. V., Whittington, R., Malkowicz, S. B., Schultz, D., Blank, K., Broderick, G. A., Tomaszewski, J. E., Renshaw, A. A., Kaplan, I., Beard, C. J., and Wein, A. (1998). Biochemical outcome after radical prostatectomy, external beam radiation therapy, or interstitial radiation therapy for clinically localized prostate cancer. JAMA 280, 969-974.

Dignan, M. B., Burhansstipanov, L., Hariton, J., Harjo, L., Rattler, T., Lee, R., and Mason, M. (2005). A comparison of two Native American Navigator formats: fact-to-face and telephone. Cancer Control 12(Suppl. 2), 28-33.

Dohan, D., and Schrag, D. (2005). Using navigators to improve care of underserved patients: current practices and approaches. Cancer 104, 848-855.

Espey, D. K., Paisano, R. E., and Cobb, N. (2003). Cancer Mortality Among American Indians and Alaska Natives: Regional Difference, 1994-1998, IHS Pub. No. 97-615-628. Rockville, MD: Indian Health Service.
Espey, D. K., Wu, X., Swan, J., Wiggins, C., Jim, M. A., Ward, E., Wingo, P. A., Howe, H. L., Ries, L. A., Miller, B. A., Jemal, A., Ahmed, F., Cobb, N., Kaur, J. S., and Edwards, B. K. (2007). Annual report to the nation on the status of cancer, 1975-2004, featuring cancer in American Indians and Alaska Natives. Cancer 110, 2119-2152.

Ford, J. G., Howerton, M. W., Lai, G. Y., Gary, T. L., Bolen, S., Gibbons, M. C., Tilburt, J., Baffi, C., Tanpitukpongse, T. P., Wilson, R. F., Powe, N. R., and Bass, E. B. (2008). Barriers to recruiting underrepresented populations to cancer clinical trials: a systematic review. Cancer 112, 228-242.

Freeman, H. P. (2004). A model patient navigation program: breaking down barriers to ensure that all individuals with cancer receive timely diagnosis and treatment. Oncol. Issues 44-46.

Freeman, H.P. (2006). Patient navigation: a community based strategy to reduce cancer disparities. J. Urban Health 83 , 139-141.

Freeman, H. P., Muth, B. J., and Kerner, J. F. (1995). Expanding access to cancer screening and clinical follow-up among the medically underserved. Cancer Pract. 3, 19-30.

Geiger, H. J. (2003). "Racial and ethnic disparities in diagnosis and treatment: a review of the evidence and a consideration of causes," Unequal Treatment: Confronting Racial and Ethnic Disparities in Health Care in Smedley, Stith, Nelson, eds B. D. Smedley, A. Y. Stith, and A. R. Nelson (Washington, DC: Institute of Medicine. National Academies Press), 415-454.

Guadagnolo, B. A., Molloy, K., Cina, K., Helbig, P., Reiner, M., and Petereit, D. G. (2008). Trust and satisfaction in a Northern Plains native American Cancer population: Walking Forward Program. 2008 Cancer Health Disparities Summit, Bethesda, MD.

Guadagnolo, B. A., Boylan, A., Sargent, M., Koop, D., Brunette, D., Kanekar, S., Shortbull, V., Molloy, K., and Petereit, D. G. (2011). Patient navigation for American Indians undergoing cancer treatment: utilization and impact on care delivery in a regional health care center. Cancer 117, 2754-2761.

Guadagnolo, B. A., Cina, K., Helbig, P., Molloy, K., Reiner, M., Cook, E. F., and Petereit, D. G. (2009a). Medical mistrust and less satisfaction with health care among Native Americans presenting for cancer treatment. J. Health Care Poor Underserved 20, 210-226.

Guadagnolo, B. A., Cina, K., Helbig, P., Molloy, K., Reiner, M., Cook, E. F., and Petereit, D. G. (2009b). Assessing cancer stage and screening dispari- ties among Native American cancer patients. When free primary care is not enough. Public Health Rep. 124, 79-89. Guadagnolo, B., Petereit, D., Helbig, P. Koop, D., Kussman, P., Fox, Dunn, E., and Patnaik, A (2009c). Involving American Indians and medically underserved populations in cancer clinical trials. Clin. Trials 6, 610-617.

Haverkamp, D., Espey, D., Paisano, R. and Cobb, N. (2008). Cancer Mortality among American Indians and Alaska Natives: Regional Differences, 1999 2003. Rockville, MD: Indian Health Service.

Kanekar, S., and Petereit, D. G. (2009). Walking forward: a program designed to lower cancer mortality rates among American Indians in western South Dakota. S. D. Med. 62, 151-157.

Lannin, D. R., Mathews, H. F., Mitchell, J., Swanson, M. S., Swanson, F. H., and Edwards, M. S. (1998). Influence of socioeconomic and cultural factors on racial differences in late-stage presentation of breast cancer. JAMA 279, 1801-1807.

Maurer, L. H., Davis, T., Hammond, S., Smith, E., West, P., and Doolittle, M. (2001). Clinical trials in a rural population: professional educational aspects. J. Cancer Educ. 16, 89-92.

McCloskey, S. A., Jaggernauth, W., Rigual, N. R., Hicks, W. L. Jr., Popat, S. R. Sullivan, M., Mashtare, T. L. Jr., Khan, M. K., Loree, T. R., and Singh, A. K. (2009). Radiation treatment interruptions greater than one week and low hemoglobin levels $(12 \mathrm{~g} / \mathrm{dL})$ are predictors of local regional failure after definitive concurrent chemotherapy and intensity-modulated radiation therapy for squamous cell carcinoma of the head and neck. Am. J. Clin. Oncol. 32, 587-591.

Mello, M. M., and Wolf, L. E. (2010), The Havasupai Indian tribe case lessons for research involving stored biologic samples. N. Engl. J. Med.363, 204-207.

Molloy, K., Reiner, M., Ratteree, K., Cina, K., Helbig, P., Psych, D., Miner, R., Elk, D. L., Spotted Tail, C., Sparks, S., Tiger, L., Esmond, S., and Petereit, D. G. (2007). Patient navigation and cultural competency in cancer care. Assoc. Community Cancer Centers Oncol. Issues 22, 38-41.

Murthy, V. H., Krumholz, H. M., and Gross, C. P. (2004). Participation in cancer clinical trials: race-, sex-, and age-based disparities. JAMA 291 , 2720-2726.

Nash, D.,Azeez, S., Vlahov, D., and Schori, M. (2006). Evaluation of an intervention to increase screening colonoscopy in an urban public hospital setting. $J$. Urban Health 83, 231-243.
Nattinger, A. B., Gottlieb, M. S., Veum, J., Yahnke, D., and Goodwin, J. S. (1992). Geographic variation in the use of breast-conserving treatment for breast cancer. N. Engl. J. Med. 326, 1102-1107.

Pandhi, N., Guadagnolo, B. A., Kanekar, S., Petereit, D. G., Karki, C., and Smith, M. A. (2010a). Intention to receive cancer screening in Native Americans from the Northern Plains. Cancer Causes Control 22, 199-206.

Pandhi, N., Guadagnolo, B. A., Kanekar, S., Petereit, D. G., and Smith, M. A. (2010b). Cancer screening in Native Americans from the Northern Plains. Am. J. Prev. Med. 38, 389-395.

Petereit, D. G., and Burhansstipanov, L. (2008). Establishing trusting partnerships for successful recruitment of American Indians to clinical trials. Cancer Control 15, 260-268.

Petereit, D. G., Molloy, K., Reiner, M., Helbig, P., Cina, K., Miner, R., Spotted Tail, C., Rost, C., Conroy, P., and Roberts, C. R. (2008). Establishing a patient navigator program to reduce cancer disparities in the American Indian communities of Western, South Dakota. Cancer Control 15, 254-259.

Petereit, D. G., Moser, A., Hahn, J., Boylan, A., Kanekar, S., Ritter, M., Bentzen, S., Koop, D., Kaur, J., and Mehta, M. (2010). Ataxia telangiectasia mutated (ATM) gene variants in American Indians. Int. J. Radiat. Oncol. Biol. Phys. 78, S90.

Petereit, D. G., Rogers, D., Govern, F., Coleman, N., Osburn, C. H., Howard, S. P., Kaur, J., Burhansstipanov, L., Fowler, C. J., Chappell, R., and Mehta, M.P. (2004). Increasing access to clinical cancer trials and emerging technologies for minority populations: the Native American Project. J. Clin. Oncol. 22, 4452-4455.

Petereit, D. G., Sarkaria, J. N., Hartmann, T. J., Kinsella, T. J., Stitt, J. A. Thomadsen, B. R., and Buchler, D. A. (1995). The adverse effect of treatment prolongation in cervical carcinoma. Int. J. Radiat. Oncol. Biol. Phys. 32, 1301-1307.

Rades, D., Stoehr, M., Kazic, N., Hakim, S. G., Walz, A., Schild, S. E., and Dunst, J. (2008). Locally advanced stage IV squamous cell carcinoma of the head and neck: impact of pre-radiotherapy hemoglobin level and interruptions during radiotherapy. Int. J. Radiat. Oncol. Biol. Phys. 70, 1108-1114.

Reynolds, S. (2010). The Cancer Expert Corps: An Idea for Building Human Capital. NCI Cancer Bulletin. Available at: http://www.cancer.gov/ncicancerbulletin/113010/page4 7

Ritter, M. A., Forman, J. D., Patrick Kupelian, M. D., Colleen Lawton, M. 
D., and Daniel Petereit, M. D. (2009). A phase I/II trial of increasingly hypofractionated radiation therapy for prostate cancer. American Society of Therapeutic Radiation Oncology 2009 Annual Meeting, Chicao, IL.

Rogers, D., and Petereit, D. G. (2005). Cancer disparities research partnership in Lakota Country: clinical trials, patient services and community education for the Oglala, Rosebud and Cheyenne River Sioux tribes. Am. J. Public Health 95, 1-4.

Sateren, W. B., Trimble, E. L., Abrams, J., Brawley, O., Breen, N., Ford, L., McCabe, M., Kaplan, R., Smith, M., Ungerleider, R., and Christian, M. C. (2002). How sociodemographics, presence of oncology specialists, and hospital cancer programs affect accrual to cancer treatment trials. J. Clin. Oncol. 20, 2109-2117.

Shavers, V. L., and Brown, M. L. (2002). Racial and ethnic disparities in the receipt of cancer treatment. J. Natl. Cancer Inst. 94, 334-357.

Stewart, J. H., Bertoni, A. G., Staten, J. L., Levine, E. A., and Gross, C. P. (2007). Participation in surgical oncology clinical trials: gender-, race/ethnicity-, and age-based disparities. Ann. Surg. Oncol. 14, 3328-3334.

Subrahmanian, K., Petereit, D. G., Kanekar, S., Burhansstipanov, L., Esmond, S., Miner, R., Spotted Tail, C., and Guadagnolo, B. A. (2011). Community-based participatory development, implementation, and evaluation of a cancer screening educational intervention among American Indians in the Northern Plains. J. CancerEduc. doi: 10.1007/s13187-0110211-5.[Epub ahead of print].

Ward, E., Jemal, A., Cokkinides, V., Singh, G. K., Cardinez, C., Ghafoor, A., and Thun, M. (2004). Cancer disparities by race/ethnicity and socioeconomic status. CA Cancer J. Clin. 54, 278-293.
Weinrich, S. P., Boyd, M. D., Weinrich, M., Greene, F., Reynolds, W. A. Jr., and Metlin, C. (1998). Increasing prostate cancer screening in African American men with peer-educator and clientnavigator interventions. J. Cancer Educ. 13, 213-219.

Wells, K. J., Battaglia, T. A., Dudley, D. J., Garcia, R., Greene, A., Calhoun, E., Mandelblatt, J. S., Paskett, E. D., Raich, P.C., and Patient Navigation Research Program. (2008). Patient navigation: state of the art or is it science? Cancer 113, 1999-2010.

Wennberg, J. E. (1996). "Breast sparing surgery," in Sciences TDMSCfEC: The Dartmouth Atlas of Health Care, eds J. E. Wennberg and M. M. Cooper (Chicago, IL: American Hospital Publishing), 128-129.

Conflict of Interest Statement: The authors declare that the research was conducted in the absence of any commercial or financial relationships that could be construed as a potential conflict of interest.

Received: 22 March 2011; accepted: 09 June 2011; published online: 22 June 2011.

Citation: Petereit DG, Guadagnolo $B A$, Wong $R$ and Coleman CN (2011) Addressing cancer disparities among American Indians through innovative technologies and patient navigation: the walking forward experience. Front. Oncol. 1:11. doi: 10.3389/fonc.2011.00011

This article was submitted to Frontiers in Radiation Oncology, a specialty of Frontiers in Oncology.

Copyright (c) 2011 Petereit, Guadagnolo, Wong and Coleman. This is an open-access article subject to a non-exclusive license between the authors and Frontiers Media $S A$, which permits use, distribution and reproduction in other forums, provided the original authors and source are credited and other Frontiers conditions are complied with. 